\title{
Synthesis and Biological Assessment of Carbazole Linked Pyrazole Schiff bases and Diarylthiourea Derivatives
}

\author{
1 Department of Chemistry, A. S. C. College Rahata, Ahmednagar, MS 423107, India \\ 2 Padmashri Vikhe Patil College of Arts, Science and Commerce, Pravaranagar, MS, 413713, India \\ 3 Department of Chemistry, A. C. S. College Satral, Rahuri, Ahmednagar, MS 413711, India \\ * Corresponding author's e-mail address: vijaykadnor11@gmail.com
}

RECEIVED: September 30, 2020 * REVISED: January 22, 2021 * ACCEPTED: January 25, 2021

Abstract: In this study, (E)-9-ethyl-N-((1,3-diphenyl-1H-pyrazol-4-ylmethylene)-9H-carbazol-3-amine (3a-f) and 1-(9-ethyl-9H-carbazol-6-yl)-3phenylthiourea (5a-f) derivatives were synthesized and their in vitro antimicrobial and antimalarial activities were evaluated. The structures of the synthesized compounds were elucidated and confirmed by using IR, ${ }^{1} \mathrm{H} N M R,{ }^{13} \mathrm{C} \mathrm{NMR}$, and mass spectra.

Keywords: antimicrobial, antimalarial activities, carbazole, diarylthiourea, Schiff base.

\section{INTRODUCTION}

$\mathbf{T}$ HE carbazole skeleton is a key structural motif possesses desirable electronic and charge-transport properties as well as large $\pi$ conjugated system. Due to this, the various functional groups are easily introduced into the structurally rigid carbazolyl ring. These characteristics result in the extensive potential applications of carbazolebased derivatives in the field of medicinal, ${ }^{[1-5]}$ organic $^{[6,7]}$ and material chemistry. ${ }^{[8,9]}$

Aminocarbazoles and its derivatives have gained much attraction due to their prominent biological activities. ${ }^{[10-15]}$ They have been identified as $\mathrm{Bcl}-2$ protein inhibitors ${ }^{[16]}$ NPY5 antagonists ${ }^{[17]}$ and anion receptors. ${ }^{[18]}$ They are also useful intermediates for the synthesis of various amino derivatives, dyes and pigments, stabilizers for polymers, pesticides, photographic materials and diagnostic reagents in cytochemical studies. ${ }^{[19]}$

Pyrazole derivatives represent one of the most active class of compounds and possess a wide spectrum of biological activities. ${ }^{[20-25]}$ Schiff bases have also been shown a broad range of biological activities, including antifungal, antibacterial, antimalarial, anti-inflammatory, antiviral, and antipyretic properties. ${ }^{[26,27]}$ The new tacrine carbazole hybrids having imines moiety reported to possess multifunctional agents for the treatment of $A D$ and potent activity against $A C h E$ inhibitory and antioxidant action. ${ }^{[28,29]}$ Although some drugs containing pyrazole and Schiff bases exhibit antiviral activities, ${ }^{[30,31]}$ carbazole based diarylthiourea, pyridopyrimidine-substituted urea and thiourea derivatives have been reported as potent anticancer agents ${ }^{[32]}$ and polyphenol oxidase inhibitors. ${ }^{[33]}$ Inhibitory activities of carbazole-linked urea and thiourea derivatives on lipopolysaccharide-induced NO production have been also reported. ${ }^{[34]}$ Also $p$-nitrodiarylthiourea derivative contains carbazole core have been evaluated on breast (MCF-7, T-47D, MDA-MB-453) and prostate (DU-145, PC-3, LNCaP) cancer cell lines. ${ }^{[35]}$

Based on the above information, we inferred that carbazole frame linked at 4-position in the pyrazole ring and aryl thiourea core could lead to significant increase in the potency and improve physicochemical properties. 


\section{RESULTS AND DISCUSSION}

\section{Chemistry}

The synthetic route is shown in schemes $\mathbf{1}$ and $\mathbf{2}$. Carbazole Schiff bases (3a-f) were prepared in accordance with the literature procedure, ${ }^{[36]}$ starting from the condensation of 9-ethyl-9H-carbazol-3-amine 1 with substituted 1,3-diaryl pyrazole aldehyde $\mathbf{2}$ in ethanol and a catalytic amount of glacial acetic acid. The substituted 1,3-diaryl pyrazole aldehydes were synthesized by the well-known VilsmeierHaack formylation reaction. ${ }^{[37]}$ Carbazole-based thiourea derivatives $(\mathbf{5 a} \mathbf{a} \mathbf{f})$ were synthesized by the reaction of 9-ethyl-9H-carbazol-3-amine 1 with various substituted phenylisothiocynate $\mathbf{4}$ in ethanol. The synthesis of compound $\mathbf{5 e}$ has been previously reported. ${ }^{[38]}$

The formation of carbazole linked Schiff bases (3a-f) were confirmed by various spectral methods. The FT-IR spectra clearly indicates that the IR band at $1596-1599 \mathrm{~cm}^{-1}$ appeared due to the typical azomethine $(-\mathrm{CH}=\mathrm{N})$ group and, thus, confirmed the formation of carbazole Schiff bases. Also the bands due to $-\mathrm{NH}_{2}$ group of $\mathrm{N}$-alkylated carbazolyl amine at $3390 \mathrm{~cm}^{-1}$ and the carbonyl $(\mathrm{C}=\mathrm{O})$ group of pyrazole aldehyde at $1890 \mathrm{~cm}^{-1}$ completely disappeared.
In ${ }^{1} \mathrm{H}$ NMR spectra, aldehyde $(\mathrm{CHO})$ and $\mathrm{NH}_{2}$ proton of amine peaks expected at $\delta 9.89$ and 6.55 disappeared. In the ${ }^{1} \mathrm{H}$ NMR spectra of representative compound $\mathbf{3 f}$, one new singlet that appeared at $\delta 9.18$ due to the $\mathrm{CH}=\mathrm{N}$ of azomethine confirmed the formation of Schiff bases. Similarly, in the ${ }^{13} \mathrm{CNMR}$, carbonyl group ( $\mathrm{CHO}$ ) expected at $\delta 190$ disappeared. The formation of Schiff base was confirmed by observing $(-\mathrm{CH}=\mathrm{N})$ carbon atom in the range of $\delta 152.10-162.56$ for azomethine. The mass spectrum of 3f displayed a molecular ion peak at $\mathrm{m} / \mathrm{z} 447.16$ due to $(\mathrm{M}+1)^{+}$mode. Also the high resolution mass spectrum (HRMS) of compound $\mathbf{3 f}$ showed a molecular ion peak at $\mathrm{m} / \mathrm{z}$ 447.1646, which confirmed the formation of compound $3 f$.

The thiourea derivatives $(\mathbf{5 a}-\mathbf{f})$ were confirmed by the absence of characteristic IR absorption band at 2000$2200 \mathrm{~cm}^{-1}$ ( $\mathrm{N}=\mathrm{C}=\mathrm{S}$ group). The IR band at $3100-3370 \mathrm{~cm}^{-1}$ appeared due to $\mathrm{NH}$ stretching, while the characteristic region of the high frequency for thiocarbonyl $(C=S)$ in the aromatics thioureas appeared at $1530-1549 \mathrm{~cm}^{-1}$. For example, the ${ }^{1} \mathrm{H}$ NMR spectrum of compound $\mathbf{5 a}$ showed two characteristic downfield singlets at $\delta 9.65$ and 9.81 assigned to the protons of two $\mathrm{NH}$ groups, while in ${ }^{13} \mathrm{C}$ NMR spectrum, the peak due to thiocarbonyl group in thiourea moiety of $\mathbf{5 a}$ was observed at $\delta 180.76$ because thiocarbonyl<smiles>CCn1c2ccccc2c2cc(N)ccc21</smiles><smiles>O=Cc1cn(-c2ccccc2)nc1[Al]</smiles>

\section{$\underset{\mathrm{AcOH}(\mathrm{cat}),}{\longrightarrow}$ reflux}<smiles>CCn1c2ccccc2c2cc(/N=C/c3cn(-c4ccccc4)nc3[Al])ccc21</smiles>

$\mathrm{Ar}=$ 3a<smiles>Cc1ccccc1</smiles><smiles>Cc1ccc(F)cc1</smiles><smiles>Cc1ccc(Cl)cc1</smiles><smiles>Cc1ccc(Br)cc1</smiles>

$3 e$<smiles>Cc1ccc(I)cc1</smiles><smiles>Ic1cccs1</smiles>

Scheme 1. Synthetic route of carbazole linked pyrazole Schiff base (3a-f)<smiles>CCn1c2ccccc2c2cc(N)ccc21</smiles>

1<smiles>[R]c1ccc(N=C=S)cc1</smiles>

4a-f

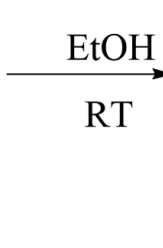<smiles>[R]c1ccc(NC(=S)Nc2ccc3c(c2)c2ccccc2n3CC)cc1</smiles>

5a-f

$$
\begin{array}{ll}
\text { 5a: } R=H, & 5 b: R=F, \\
\text { 5c: } R=C l, & 5 d: R=B r, \\
\text { 5e: } R=\text { NO }_{2}, & 5 f: R=C_{3}
\end{array}
$$

Scheme 2. Synthetic route of carbazole diarylthiourea derivative (5a-f). 
Table 1. Antimicrobial activities of the synthesized compounds 3a-f against pathological organisms expressed as minimal inhibition concentration (MIC, $\mu \mathrm{g} / \mathrm{mL}$ )

\begin{tabular}{|c|c|c|c|c|c|c|c|}
\hline \multirow{3}{*}{$\begin{array}{l}\text { Compd. } \\
\text { No. }\end{array}$} & \multicolumn{7}{|c|}{ Microorganisms } \\
\hline & \multicolumn{2}{|c|}{ Gram - ve bacteria } & \multicolumn{2}{|c|}{ Gram + ve bacteria } & \multicolumn{3}{|c|}{ Fungi } \\
\hline & $\begin{array}{c}\text { Escherichia } \\
\text { Coli }\end{array}$ & $\begin{array}{l}\text { Proteus } \\
\text { mirabilis }\end{array}$ & $\begin{array}{c}\text { Streptococcus } \\
\text { faecalis }\end{array}$ & $\begin{array}{l}\text { Bacillus } \\
\text { subtilis }\end{array}$ & $\begin{array}{l}\text { Candida } \\
\text { albicans }\end{array}$ & $\begin{array}{l}\text { Penicillium } \\
\text { chrysogenum }\end{array}$ & $\begin{array}{c}\text { Aspergillus } \\
\text { niger }\end{array}$ \\
\hline $3 a$ & 25 & 25 & 25 & 20 & 20 & 25 & 25 \\
\hline $3 b$ & 10.5 & 20 & 11.5 & 10.5 & 12.5 & 20.5 & 13.5 \\
\hline $3 c$ & 12.5 & 25 & 15.5 & 13.5 & 14.5 & 20 & 15.5 \\
\hline $3 d$ & 15 & 25 & 20 & 15.5 & 20 & 15.5 & 15 \\
\hline $3 e$ & 20.5 & 25 & 20 & 20.5 & 20 & 20.5 & 20 \\
\hline $3 f$ & 11.5 & 25 & 14.5 & 14.5 & 10.5 & 20 & 15.5 \\
\hline Streptomycin & 6.25 & 6.25 & 6.25 & 6.25 & - & - & - \\
\hline Fluconazole & - & - & - & - & 6.25 & 6.25 & 6.25 \\
\hline
\end{tabular}

group have a greater magnetic anisotropy compared to the carbonyl group. Furthermore, the mass spectrum shows the molecular ion peak at $(\mathrm{m} / \mathrm{z}) 346.13(\mathrm{M}+\mathrm{H})^{+}$which confirmed the formation of $\mathbf{5 a}$.

\section{Antibacterial and Antifungal Evaluations}

The antimicrobial activity of the synthesized compounds $\mathbf{3}$ and $\mathbf{5}$ was evaluated against two gram-negative ( $E$. coli, P. mirabilis), two gram-positive (S. faecalis, B. subtilis) bacterial strains, and three (C. albicans, $P$. chrysogenum, $A$. niger) fungal strains using streptomycin and fluconazole as a standard drugs. The minimal inhibitory concentration (MIC) values of carbazole linked pyrazole Schiff base derivatives were presented in Table 1.

It was found that among the $\mathbf{3 a}-\mathbf{f}$ derivatives $\mathbf{3 b} \mathbf{3} \mathbf{3 c}$, 3d and $\mathbf{3 f}$ showed stronger antibacterial efficacies and broader bioactive spectrum against E. coli, S. faecalis, and B. subtilis with the MIC values in the range of 10.5-15.5 $\mu \mathrm{g} / \mathrm{mL}$ comparable to that of the positive control.
Compounds $\mathbf{3 a}$ and $\mathbf{3 e}$ exhibited moderate to good inhibitory activities ( 20 and $25 \mu \mathrm{g} / \mathrm{mL}$ ) against all tested bacterial strains. Furthermore, compounds $\mathbf{3 b}, \mathbf{3 c}$ and $\mathbf{3 f}$ showed a broad spectrum of antifungal activities (10.5$15.5 \mu \mathrm{g} / \mathrm{mL}$ ) against $C$. albicans and $A$. niger as compared with standard drug fluconazole, while compounds $\mathbf{3 a}, \mathbf{3 d}$, $3 e$ and $3 f$ showed satisfactory activities $(20-25 \mu \mathrm{g} / \mathrm{mL})$.

MIC values of carbazole based thiourea derivatives (5a-f) as shown in Table 2 revealed that compounds $\mathbf{5 b}$ and 5e exhibited highest inhibition activities $(8.5-12.5 \mu \mathrm{g} / \mathrm{mL})$ against E. coli, S. faecalis and B. subtilis bacterial strains. Compounds 5b, 5c, 5d and $\mathbf{5 e}$ displayed remarkable inhibition activities with a MIC value ranging from 10.5 to $12.5 \mu \mathrm{g} / \mathrm{mL}$ against $P$. chrysogenum fungal strains, while compounds $\mathbf{5 a - f}$ showed to be less active on C. albicans and A. niger fungal strains relative to standard drug fluconazole. Most of the compounds $\mathbf{3}$ and $\mathbf{5}$ did not inhibit the growth of $P$. mirabilis bacterial strain satisfactorily, as compared with positive control streptomycin.

Table 2. Antimicrobial activities of the synthesized compounds 5a-f against pathological organisms expressed as minimal inhibition concentration (MIC, $\mu \mathrm{g} / \mathrm{mL}$ )

\begin{tabular}{|c|c|c|c|c|c|c|c|}
\hline \multirow{3}{*}{$\begin{array}{c}\text { Compd. } \\
\text { No. }\end{array}$} & \multicolumn{7}{|c|}{ Microorganisms } \\
\hline & \multicolumn{2}{|c|}{ Gram - ve bacteria } & \multicolumn{2}{|c|}{ Gram + ve bacteria } & \multicolumn{3}{|c|}{ Fungi } \\
\hline & $\begin{array}{c}\text { Escherichia } \\
\text { coli }\end{array}$ & $\begin{array}{l}\text { Proteus } \\
\text { mirabilis }\end{array}$ & $\begin{array}{c}\text { Streptococus } \\
\text { faecalis }\end{array}$ & $\begin{array}{l}\text { Bacillus } \\
\text { subtilis }\end{array}$ & $\begin{array}{l}\text { Candida } \\
\text { albicans }\end{array}$ & $\begin{array}{l}\text { Penicillium } \\
\text { chrysogenum }\end{array}$ & $\begin{array}{c}\text { Aspergillus } \\
\text { niger }\end{array}$ \\
\hline $5 a$ & 20 & 25 & 25 & 20 & 20 & 20 & 25 \\
\hline $5 b$ & 9.5 & 25 & 10.5 & 12.5 & 25 & 10.5 & 20 \\
\hline $5 c$ & 10 & 20 & 12.5 & 20 & 20 & 12.5 & 20 \\
\hline $5 d$ & 15 & 25 & 15.5 & 20 & 25 & 12.5 & 25 \\
\hline $5 e$ & 8.5 & 20 & 10.5 & 12.5 & 20 & 11.5 & 20 \\
\hline $5 f$ & 20 & 25 & 20 & 25 & 20 & 20 & 25 \\
\hline Streptomycin & 6.25 & 6.25 & 6.25 & 6.25 & - & - & - \\
\hline Fluconazole & - & - & - & - & 6.25 & 6.25 & 6.25 \\
\hline
\end{tabular}


Table 3. Antimalarial activities of the synthesized compounds $3 a-f$ and $5 a-f$ against $P$. falciparum expressed as $I C_{50}$ $(\mu \mathrm{g} / \mathrm{mL})$

\begin{tabular}{|c|c|c|c|}
\hline \multicolumn{3}{|c|}{ P. falciparum } & \multirow{2}{*}{$\begin{array}{l}\text { P. falciparum } \\
\mathrm{IC}_{50}(\mu \mathrm{g} / \mathrm{mL})\end{array}$} \\
\hline Compd. No. & $\mathrm{IC}_{50}(\mu \mathrm{g} / \mathrm{mL})$ & Compd. No. & \\
\hline $3 a$ & 1.50 & $5 a$ & 1.65 \\
\hline $3 b$ & 0.90 & $5 b$ & 0.95 \\
\hline $3 c$ & 1.00 & $5 c$ & 1.10 \\
\hline $3 d$ & 1.15 & $5 d$ & 1.25 \\
\hline $3 e$ & 1.55 & $5 e$ & 1.00 \\
\hline $3 f$ & 0.95 & $5 f$ & 1.75 \\
\hline Quinine & 0.268 & - & 0.268 \\
\hline Chloroquine & 0.020 & - & 0.020 \\
\hline
\end{tabular}

\section{Antimalarial Evaluation}

The synthesized compounds $\mathbf{3}$ and $\mathbf{5}$ were also screened for their in vitro antimalarial activity against Plasmodium falciparum strain using chloroquine and quinine as reference drugs. The mean $\mathrm{IC}_{50}(\mu \mathrm{g} / \mathrm{mL})$ values of test compounds against the test microbe are presented in Table 3. The results revealed that the majority of the synthesized compounds showed significant degrees of inhibition against $P$. falciparum as compared with standard drugs.

Among the carbazole linked pyrazole Schiff bases (3a-f), compound $\mathbf{3 b}$ with fluorine substituent attached exhibited a respectable antimalarial spectrum with $\mathrm{IC}_{50}$ value of $0.90 \mu \mathrm{g} / \mathrm{mL}$, as compared with compounds $\mathbf{3 a}, \mathbf{3 c}$, $\mathbf{3 d}$ and $\mathbf{3 e}$ and standard drug quinine. Compounds $\mathbf{3 a}, \mathbf{3 c}$, $\mathbf{3 d}$ and $\mathbf{3 e}$ exhibited satisfactory antimalarial activity with $\mathrm{IC}_{50}$ value ranging from 1.00 to $1.55 \mu \mathrm{g} / \mathrm{mL}$. Compound $\mathbf{3 f}$ with thiophene substituent showed considerable inhibition activities with $\mathrm{IC}_{50}$ value of $0.95 \mu \mathrm{g} / \mathrm{mL}$. In case of carbazole bearing diarylthiourea derivatives ( $\mathbf{5 a}-\mathbf{f}), \mathbf{5 b}$ and $\mathbf{5 e}$ showed the strong growth inhibition activities (with $\mathrm{IC}_{50}$ values of 0.95 and $1.00 \mu \mathrm{g} / \mathrm{mL}$ ) as compared with standard drug quinine, while compounds $\mathbf{5 a}, \mathbf{5 c}, \mathbf{5 d}$ and $\mathbf{5 f}$ showed moderate to good inhibition activities against $P$. falciparum comparable to that of the reference compounds.

\section{CONCLUSIONS}

To summarize, syntheses of a series of $(E)-9$-ethyl- $N-((1,3-$ diphenyl-1H-pyrazol-4-yl)methylene)-9H-carbazol-3-amine (3a-f) and 1-(9-ethyl-9H-carbazol-6-yl)-3-phenylthiourea (5a-f) were carried out in which some electron withdrawing, donating as well as bulky functional groups were introduced to the phenyl ring. Also, it is known that the presence of halides $(\mathrm{F}, \mathrm{Cl}, \mathrm{Br})$ increases the lipophilic properties of the compounds. Antimicrobial study of the synthesized compounds indicated that some of the compounds displayed moderate to potent antibacterial and antifungal activities which will be further helpful for the development of new antimicrobial compounds. As structure-activity relationship (SAR) study of all the compounds was taken into account (Figure 1), it was observed that the
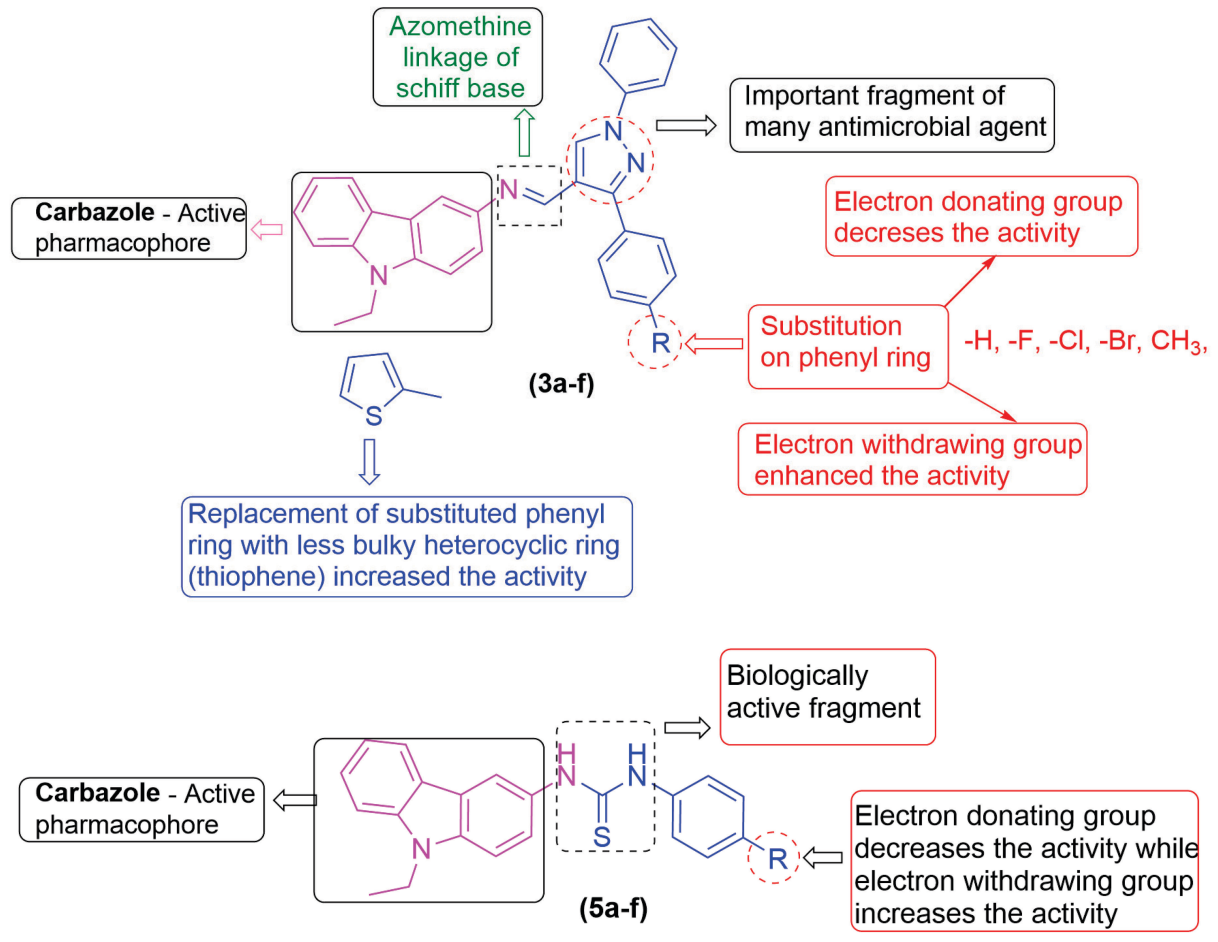

Figure 1 . Structure-activity relationship of target compounds 3 and 5. 
combination of carbazole moiety to various pyrazole aldehyde and substituted phenylisothiocynate in new derivatives caused increased activities against most of the tested organisms. The results also suggested that the antimicrobial activities of the carbazole derivatives were influenced by the aromatic substituent. Compounds with electron-withdrawing substituent $\mathbf{3 b}, \mathbf{3 c}, \mathbf{3 d}, \mathbf{5 b}, \mathbf{5 c}, \mathbf{5 d}$ and $\mathbf{5 e}$ in the phenyl ring were more potent against most of the tested microorganisms than compounds $\mathbf{3 e}$ and $\mathbf{5 f}$ with electron-donating groups. Furthermore, compounds $3 \mathbf{3}$ and $5 \mathbf{a}$ without substituent in the phenyl ring showed satisfactory activities against all tested bacterial and fungal strains. It was observed that, the sulphur containing Schiff base and diarylthiourea derivatives showed remarkably increments in the antibacterial, antifungal and antimalarial activities. In case of diarylthiourea derivatives, compound 5b with fluorine substituent increased the antimalarial activity of the molecule, as compared with compounds containing chlorine, bromine and methyl groups. High potency and promising antimicrobial activity of the synthesized compounds $\mathbf{3} \mathbf{a}-\mathbf{f}$ and $\mathbf{5 a}-\mathbf{f}$ suggest that these compounds could serve as good leads for further optimization and development.

\section{EXPERIMENTAL}

The recorded melting points were determined in an open capillary and are uncorrected. IR spectra were recorded on Perkin Elmer Fourier-transform infrared (FTIR) spectrophotometer with ATR. The ${ }^{1} \mathrm{H}$ NMR and ${ }^{13} \mathrm{C}$ NMR spectra were recorded on a Bruker Avance II $(500 \mathrm{MHz})$ and Bruker (125 MHz) spectrometer respectively, using TMS as internal standard. Mass spectra were recorded on a Waters Q-TOF micromass spectrophotometer. The thin layer chromatography (TLC) was carried out on precoated silica gel aluminum plates to check compounds purity. The starting materials 9-ethyl-9H-carbazol-3-amine and substituted phenylisothiocynate of Sigma Aldrich make were purchased from local chemical providers. The antimicrobial activity was evaluated by the agar well diffusion method. ${ }^{[39]}$ The in vitro antimalarial assay was carried out in 96 well plates according to the micro assay protocol of Rieckmann, and co-workers with minor modifications. ${ }^{[40]}$

\section{Minimal Inhibitory Concentration (MIC) Measurement}

The potato dextrose broths used for microorganism susceptibility tests in nutrient media for the determination of MIC. The tested compound stock $1000 \mu \mathrm{g} / \mathrm{mL}$ solutions, Streptomycin and Fluconazole were prepared in DMSO followed by dilutions to $100-6.25 \mu \mathrm{g} / \mathrm{mL}$ concentrations. Inoculated microorganism suspensions were incubated at $37^{\circ} \mathrm{C}$ for $1-5$ days for MIC determination.

\section{General Procedure for the Synthesis of Compounds 3a-f}

9-Ethyl-9H-carbazol-3-amine 1 (1.5 mmol) and substituted 1,3-diphenyl-1H-pyrazole-4-carbaldehyde 2 ( $1.5 \mathrm{mmol}$ ) were dissolved in ethanol $(10 \mathrm{~mL})$ and refluxed in reaction flask for $1 \mathrm{~h}$. After that, 2-3 drops of acetic acid was added to solvent as a catalyst. As a result of condensation reaction reddish brown color was observed. After completion of the reaction (monitored by TLC), the reaction mixture was cooled and light yellow colored spongy solid separated. The obtained solid was filtered and purified by recrystallization from DMF to afford compounds 3a-f.

(E)-9-ethyl- $N$-((1,3-diphenyl-1H-pyrazol-4-yl)methylene)9H-carbazol-3-amine (3a) Yellow colored solid; Yield (71 \%); m.p. $124-125^{\circ} \mathrm{C}$; IR $\tilde{v}_{\max } / \mathrm{cm}^{-1}$ : $3060(=\mathrm{CH}), 1599(-\mathrm{CH}=\mathrm{N})$, $1504(\mathrm{C}=\mathrm{C}) ;{ }^{1} \mathrm{H}$ NMR $\left(\mathrm{CDCl}_{3}\right) \delta / \mathrm{ppm}: 1.42(\mathrm{t}, 3 \mathrm{H}, J=7.1 \mathrm{~Hz}$, $\left.\mathrm{CH}_{3}\right), 4.41\left(\mathrm{q}, 2 \mathrm{H}, J=7.1 \mathrm{~Hz}, \mathrm{~N}-\mathrm{CH}_{2}\right), 7.20-7.26(\mathrm{~m}, 1 \mathrm{H}, \mathrm{ArH})$, 7.30-7.35 (m, 1H, ArH), 7.41-7.48 (m, 3H, ArH), 7.50-7.55 (m, 5H, ArH), 7.60-7.75 (m, 5H, ArH), 7.85-8.10 (m, 2H, $\mathrm{ArH}), 8.75(\mathrm{~s}, 1 \mathrm{H},-\mathrm{CH}=\mathrm{N}), 8.89\left(\mathrm{~s}, 1 \mathrm{H}\right.$, pyrazole ring); ${ }^{13} \mathrm{C}$ $\mathrm{NMR}\left(\mathrm{CDCl}_{3}\right) \delta / \mathrm{ppm}: 13.80,37.75,108.71,108.80,112.38$, $118.83,119.42,119.71,120.65,120.73,122.98,123.55$, $125.99,127.38,127.50,129.64,130.42,131.45,131.90$, 138.70, 139.55, 140.59, 143.90, 150.15, 152.59; MS (m/z): $441.05(\mathrm{M}+\mathrm{H})^{+}$.

(E)-9-ethyl-N-((3-(4-fluorophenyl)-1-phenyl-1H-pyrazol-4yl)methylene)-9H-carbazol-3-amine (3b) Yellow colored solid; Yield (67\%); m.p. $116-117{ }^{\circ} \mathrm{C}$; IR $\tilde{v}_{\max } / \mathrm{cm}^{-1}: 3048$ $(=\mathrm{CH}), 1596(-\mathrm{CH}=\mathrm{N}), 1504(\mathrm{C}=\mathrm{C}), 1216(\mathrm{Ar}-\mathrm{F}) ;{ }^{1} \mathrm{H} \mathrm{NMR}$ $\left(\mathrm{CDCl}_{3}\right) \delta / \mathrm{ppm}: 1.42\left(\mathrm{t}, 3 \mathrm{H}, J=7.0 \mathrm{~Hz}, \mathrm{CH}_{3}\right), 4.42$ (q, $2 \mathrm{H}$, $\left.J=7.0 \mathrm{~Hz}, \mathrm{~N}-\mathrm{CH}_{2}\right), 7.20-7.29(\mathrm{~m}, 1 \mathrm{H}, \mathrm{ArH}), 7.38-7.45(\mathrm{~m}$, $1 \mathrm{H}, \mathrm{ArH}), 7.49-7.56(\mathrm{~m}, 3 \mathrm{H}, \mathrm{ArH}), 7.59-7.75(\mathrm{~m}, 4 \mathrm{H}, \mathrm{ArH})$, 7.80-7.95 (m, 4H, ArH), 7.99-8.20 (m, 3H, ArH), $8.68(\mathrm{~s}, 1 \mathrm{H}$, $-\mathrm{CH}=\mathrm{N}), 8.75\left(\mathrm{~s}, 1 \mathrm{H}\right.$, pyrazole ring); ${ }^{13} \mathrm{C} \mathrm{NMR}\left(\mathrm{CDCl}_{3}\right) \delta / \mathrm{ppm}$ : $13.85,37.70,108.72,108.85,112.49,118.90,119.47,119.70$, $120.68,120.79,122.95,123.59,125.90,127.36,127.50$, $129.60,130.45,131.47,131.90,138.70,139.53,140.55$, 143.92, 150.10, 152.66; MS (m/z): $459.10(\mathrm{M}+\mathrm{H})^{+}$.

(E)-N-((3-(4-chlorophenyl)-1-phenyl-1H-pyrazol-4-yl)methylene)-9-ethyl-9H-carbazol-3-amine (3c) Yellow colored solid; Yield (70 \%); m.p. 138-139 ${ }^{\circ} \mathrm{C}$; IR $\tilde{v}_{\max } / \mathrm{cm}^{-1}$ : $3048(=\mathrm{CH})$, $1598(-\mathrm{CH}=\mathrm{N}), 1503(\mathrm{C}=\mathrm{C}), 1089(\mathrm{Ar}-\mathrm{Cl}) ;{ }^{1} \mathrm{H} \mathrm{NMR}\left(\mathrm{CDCl}_{3}\right)$ $\delta / \mathrm{ppm}: 1.44\left(\mathrm{t}, 3 \mathrm{H}, J=7.1 \mathrm{~Hz}, \mathrm{CH}_{3}\right), 4.37(\mathrm{q}, 2 \mathrm{H}, J=7.1 \mathrm{~Hz}$, $\left.\mathrm{N}-\mathrm{CH}_{2}\right), 7.21-7.34(\mathrm{~m}, 2 \mathrm{H}, \mathrm{ArH}), 7.36-7.50(\mathrm{~m}, 3 \mathrm{H}, \mathrm{ArH})$, 7.52-7.77 (m, 5H, ArH), 7.81-7.85 (m, 4H, ArH), 7.89-8.13 $(\mathrm{m}, 2 \mathrm{H}, \mathrm{ArH}), 8.65(\mathrm{~s}, 1 \mathrm{H},-\mathrm{CH}=\mathrm{N}), 8.71(\mathrm{~s}, 1 \mathrm{H}$, pyrazole ring); ${ }^{13} \mathrm{C}$ NMR $\left(\mathrm{CDCl}_{3}\right) \delta / \mathrm{ppm}: 13.84,37.70,108.69,108.85$, $112.36,118.90,119.37,119.70,120.58,120.64,122.94$, $123.50,125.96,127.31,127.57,128.97,129.59,130.11$ 134.71, 138.72, 139.46, 140.53, 143.91, 150.14, 152.51, 162.56; MS $(\mathrm{m} / \mathrm{z}): 475.23(\mathrm{M}+\mathrm{H})^{+}$. 
(E)-N-((3-(4-bromophenyl)-1-phenyl-1H-pyrazol-4-yl)methylene)-9-ethyl-9H-carbazol-3-amine (3d) Yellow colored solid; Yield (68 \%); m.p. 130-131 ${ }^{\circ} \mathrm{C}$; IR $\tilde{v}_{\max } / \mathrm{cm}^{-1}$ : $3051(=\mathrm{CH})$, $1598(-\mathrm{CH}=\mathrm{N}), 1504(\mathrm{C}=\mathrm{C}), 698(\mathrm{Ar}-\mathrm{Br}) ;{ }^{1} \mathrm{H} \mathrm{NMR}\left(\mathrm{CDCl}_{3}\right)$ $\delta /$ ppm: $1.44\left(\mathrm{t}, 3 \mathrm{H}, J=7.1 \mathrm{~Hz}, \mathrm{CH}_{3}\right), 4.38(\mathrm{q}, 2 \mathrm{H}, J=7.1 \mathrm{~Hz}$, $\left.\mathrm{N}-\mathrm{CH}_{2}\right), 7.21-7.25(\mathrm{~m}, 1 \mathrm{H}, \mathrm{ArH}), 7.34-7.37(\mathrm{~m}, 1 \mathrm{H}, \mathrm{ArH})$, 7.41-7.46 (m, 3H, ArH), 7.49-7.55 (m, 3H, ArH), 7.62-7.77 (m, 4H, ArH), 7. 83-7.85 (m, 2H, ArH), 7.95-8.10 (m, 2H, $\mathrm{ArH}), 8.65(\mathrm{~s}, 1 \mathrm{H},-\mathrm{CH}=\mathrm{N}), 8.96\left(\mathrm{~s}, 1 \mathrm{H}\right.$, pyrazole ring); ${ }^{13} \mathrm{C}$ $\operatorname{NMR}\left(\mathrm{CDCl}_{3}\right) \delta / \mathrm{ppm}: 13.88,37.73,108.70,108.85,112.39$, $118.93,119.40,119.74,120.63,120.70,122.99,123.54$, $125.98,127.34,127.55,129.62,130.41,131.46,131.95$, $138.75,139.50,140.56,143.96,150.11,152.56$; MS $(m / z)$ : $519.16(\mathrm{M}+\mathrm{H})^{+}$.

(E)-9-ethyl-N-((1-phenyl-3-p-tolyl-1H-pyrazol-4-yl)methylene)-9H-carbazol-3-amine (3e) Yellow colored solid; Yield (70 \%); m.p. $122-123^{\circ} \mathrm{C}$; IR $\tilde{v}_{\max } / \mathrm{cm}^{-1}$ : 3051 (=CH), 1598 $(-\mathrm{CH}=\mathrm{N}), 1531(\mathrm{C}=\mathrm{C}) ;{ }^{1} \mathrm{H} \mathrm{NMR}\left(\mathrm{CDCl}_{3}\right) \delta / \mathrm{ppm}: 1.39(\mathrm{t}, 3 \mathrm{H}$, $\left.J=7.1 \mathrm{~Hz}, \mathrm{CH}_{3}\right), 2.30\left(\mathrm{~s}, 3 \mathrm{H}, \mathrm{ArCH}_{3}\right), 4.44(\mathrm{q}, 2 \mathrm{H}, J=7.1 \mathrm{~Hz}$, $\left.\mathrm{N}-\mathrm{CH}_{2}\right), 7.20-7.27(\mathrm{~m}, 2 \mathrm{H}, \mathrm{ArH}), 7.42-7.50(\mathrm{~m}, 3 \mathrm{H}, \mathrm{ArH})$, 7.55-7.69 (m, 5H, ArH), 8.03-8.12 (m, 4H, ArH), 8.18-8.28 $(\mathrm{m}, 2 \mathrm{H}, \mathrm{ArH}), 8.89(\mathrm{~s}, 1 \mathrm{H},-\mathrm{CH}=\mathrm{N}), 9.17(\mathrm{~s}, 1 \mathrm{H}$, pyrazole ring); ${ }^{13} \mathrm{C}$ NMR $\left(\mathrm{CDCl}_{3}\right) \delta / \mathrm{ppm}: 13.84,20.89,37.78,108.65$, $108.88,112.40,118.92,119.36,119.72,119.80,120.46$, $120.73,123.13,123.58,125.97,126.50,127.10,127.38$, $127.67,127.70,129.68,134.40,138.70,139.39,140.58$, 144.60, 147.68, 150.18; MS (m/z): $455.08(\mathrm{M}+\mathrm{H})^{+}$.

(E)-9-ethyl-N-((1-phenyl-3-(thiophen-2-yl)-1H-pyrazol-4yl)methylene)-9H-carbazol-3-amine (3f) Yellow colored solid; Yield (72 \%); m.p. 109-110 ${ }^{\circ} \mathrm{C}$; IR $\tilde{v}_{\max } / \mathrm{cm}^{-1}: 3054$ $(=\mathrm{CH}), 1597(-\mathrm{CH}=\mathrm{N}), 1510(\mathrm{C}=\mathrm{C}), 1185(\mathrm{C}-\mathrm{S}) ;{ }^{1} \mathrm{H}$ NMR $\left(\mathrm{CDCl}_{3}\right) \delta / \mathrm{ppm}: 1.34\left(\mathrm{t}, 3 \mathrm{H}, J=7.1 \mathrm{~Hz}, \mathrm{CH}_{3}\right), 4.45(\mathrm{q}, 2 \mathrm{H}$, $\left.J=7.1 \mathrm{~Hz}, \mathrm{~N}-\mathrm{CH}_{2}\right), 7.19-7.23(\mathrm{~m}, 2 \mathrm{H}, \mathrm{ArH}), 7.40-7.51(\mathrm{~m}$, $3 \mathrm{H}$, thiophenen ring), 7.57-7.69 (m, 5H, ArH), 8.00-8.10 (m, $3 \mathrm{H}, \mathrm{ArH}), 8.16-8.24(\mathrm{~m}, 2 \mathrm{H}, \mathrm{ArH}), 8.88(\mathrm{~s}, 1 \mathrm{H},-\mathrm{CH}=\mathrm{N}), 9.15$ (s, $1 \mathrm{H}$, pyrazole ring); ${ }^{13} \mathrm{C} \mathrm{NMR}\left(\mathrm{CDCl}_{3}\right) \delta / \mathrm{ppm}: 13.88,37.74$, $108.69,108.85,112.43,118.91,119.37,119.71,119.82$, $120.51,120.63,123.03,123.56,125.96,126.51,127.15$, $127.28,127.65,127.75,129.58,134.42,138.75,139.38$, 140.56, 144.10, 147.69, 150.14; MS (m/z): $447.16(\mathrm{M}+\mathrm{H})^{+}$.

\section{General Procedure for the Synthesis of Compounds 5a-f}

Substituted phenylisothiocynate $4(1 \mathrm{mmol})$ was added dropwise to a well stirred solution of 9-ethyl-9H-carbazol3-amine 1 (1 mmol) in ethanol $(5 \mathrm{~mL})$, with constant stirring. A light yellow colored solid precipitated, which after $30 \mathrm{~min}$ was filtered off and crude compounds were recrystallized from ethanol to afford the target compounds $\mathbf{5 a - f}$.

1-(9-ethyl-9H-carbazol-6-yl)-3-phenylthiourea (5a) Light yellow colored solid; Yield (68\%); m.p. $158-159{ }^{\circ} \mathrm{C}$; IR $\tilde{v}_{\max } / \mathrm{cm}^{-1}$ : $3357(\mathrm{NH}), 3173(\mathrm{NH}), 2965(=\mathrm{CH}), 1534(\mathrm{C}=\mathrm{S})$; ${ }^{1} \mathrm{H} \operatorname{NMR}\left(\mathrm{CDCl}_{3}\right) \delta / \mathrm{ppm}: 1.33\left(\mathrm{t}, 3 \mathrm{H}, J=6.2 \mathrm{~Hz}, \mathrm{CH}_{3}\right), 4.45$ (q, $\left.2 \mathrm{H}, J=6.7 \mathrm{~Hz}, \mathrm{~N}-\mathrm{CH}_{2}\right), 7.12-7.22(\mathrm{~m}, 4 \mathrm{H}, \mathrm{ArH}), 7.33-7.36$ $(\mathrm{m}, 4 \mathrm{H}, \mathrm{ArH}), 7.45-7.54(\mathrm{~m}, 2 \mathrm{H}, \mathrm{ArH}), 7.59-8.15(\mathrm{~m}, 2 \mathrm{H}$, ArH), $9.65(\mathrm{~s}, 1 \mathrm{H}, \mathrm{NH}), 9.81(\mathrm{~s}, 1 \mathrm{H}, \mathrm{NH}) ;{ }^{13} \mathrm{C} \mathrm{NMR}\left(\mathrm{CDCl}_{3}\right)$ $\delta / \mathrm{ppm}: 13.87,37.78,108.87,109.40,118.79,119.39$, $120.76,122.37,123.62,124.45,125.18,126.52,126.63$, $127.68,129.24,137.76,138.99,140.58,180.76 ; \mathrm{MS}(\mathrm{m} / \mathrm{z})$ : $346.13(\mathrm{M}+\mathrm{H})^{+}$.

1-(9-ethyl-9H-carbazol-6-yl)-3-(4-fluorophenyl) thiourea (5b) Light yellow colored solid; Yield (65 \%); m.p. $161-162{ }^{\circ} \mathrm{C}$; IR $\tilde{v}_{\max } / \mathrm{cm}^{-1}: 3366(\mathrm{NH}), 3183(\mathrm{NH}), 2964(=\mathrm{CH}), 1530(\mathrm{C}=\mathrm{S})$; ${ }^{1} \mathrm{H} \mathrm{NMR}\left(\mathrm{CDCl}_{3}\right) \delta / \mathrm{ppm}: 1.44\left(\mathrm{t}, 3 \mathrm{H}, J=7.0 \mathrm{~Hz}, \mathrm{CH}_{3}\right), 4.44$ (q, $\left.2 \mathrm{H}, J=6.7 \mathrm{~Hz}, \mathrm{~N}-\mathrm{CH}_{2}\right), 7.15-7.17(\mathrm{~m}, 1 \mathrm{H}, \mathrm{ArH}), 7.20-7.28$ $(\mathrm{m}, 2 \mathrm{H}, \mathrm{ArH}), 7.32-7.37(\mathrm{~m}, 1 \mathrm{H}, \mathrm{ArH}), 7.40-7.45(\mathrm{~m}, 3 \mathrm{H}$, ArH), 7.48-7.53 (m, 3H, ArH), 7.56-7.65 (m, 1H, ArH), 8.07 $(\mathrm{s}, 1 \mathrm{H}, \mathrm{NH}), 8.21(\mathrm{~s}, 1 \mathrm{H}, \mathrm{NH}) ;{ }^{13} \mathrm{C} \mathrm{NMR}\left(\mathrm{CDCl}_{3}\right) \delta / \mathrm{ppm}: 14.28$, $37.76,110.22$, 110.29, 117.30, 120.33, 121.14, 122.47, $122.75,122.99,123.15,123.25,123.91,125.73,127.00$, $128.38,129.11,135.44,140.65,142.21,149.72,157.45$, 192.98; MS (m/z): $364.15(\mathrm{M}+\mathrm{H})^{+}$.

1-(4-chlorophenyl)-3-(9-ethyl-9H-carbazol-6-yl) thiourea (5c) Light yellow colored solid; Yield (69 \%), m.p. $121-122^{\circ} \mathrm{C}$; IR $\tilde{v}_{\max } / \mathrm{cm}^{-1}$ : $3321(\mathrm{NH}), 3120(\mathrm{NH}), 2973(=\mathrm{CH}), 1529(\mathrm{C}=\mathrm{S})$; ${ }^{1} \mathrm{H} \mathrm{NMR}\left(\mathrm{CDCl}_{3}\right) \delta / \mathrm{ppm}: 1.33\left(\mathrm{t}, 3 \mathrm{H}, J=6.2 \mathrm{~Hz}, \mathrm{CH}_{3}\right), 4.47$ (q, $\left.2 \mathrm{H}, J=6.7 \mathrm{~Hz}, \mathrm{~N}-\mathrm{CH}_{2}\right), 7.16-7.22(\mathrm{~m}, 3 \mathrm{H}, \mathrm{ArH}), 7.33-7.37$ (m, 3H, ArH), 7.43-7.49 (m, 3H, ArH), 7.60-8.17 (m, 2H, ArH), $9.75(\mathrm{~s}, 1 \mathrm{H}, \mathrm{NH}), 9.99(\mathrm{~s}, 1 \mathrm{H}, \mathrm{NH}) ;{ }^{13} \mathrm{C} \mathrm{NMR}\left(\mathrm{CDCl}_{3}\right) \delta / \mathrm{ppm}$ : $13.87,37.82$, 108.96, 109.69, 118.94, 119.56, 120.78, 122.24, $123.01,123.83,124.34,124.86,126.35,126.76,129.86$, 134.43, 139.19, 140.63, 180.63; MS (m/z): $380.04(\mathrm{M}+\mathrm{H})^{+}$

1-(4-bromophenyl)-3-(9-ethyl-9H-carbazol-6-yl)thiourea (5d) Light yellow colored solid; Yield (65 \%); m.p. $132-133^{\circ} \mathrm{C}$; IR $\tilde{v}_{\max } / \mathrm{cm}^{-1}: 3330(\mathrm{NH}), 3128(\mathrm{NH}), 2975(=\mathrm{CH}), 1525(\mathrm{C}=\mathrm{S})$; ${ }^{1} \mathrm{H} \mathrm{NMR}\left(\mathrm{CDCl}_{3}\right) \delta / \mathrm{ppm}: 1.34\left(\mathrm{t}, 3 \mathrm{H}, J=6.4 \mathrm{~Hz}, \mathrm{CH}_{3}\right), 4.45$ (q, $\left.2 \mathrm{H}, J=6.8 \mathrm{~Hz}, \mathrm{~N}-\mathrm{CH}_{2}\right), 7.18-7.25(\mathrm{~m}, 3 \mathrm{H}, \mathrm{ArH}), 7.32-7.39$ $(\mathrm{m}, 3 \mathrm{H}, \mathrm{ArH}), 7.45-7.55(\mathrm{~m}, 3 \mathrm{H}, \mathrm{ArH}), 7.65-8.10(\mathrm{~m}, 2 \mathrm{H}$, $\mathrm{ArH}), 9.60(\mathrm{~s}, 1 \mathrm{H}, \mathrm{NH}), 9.70(\mathrm{~s}, 1 \mathrm{H}, \mathrm{NH}) ;{ }^{13} \mathrm{C} \mathrm{NMR}\left(\mathrm{CDCl}_{3}\right)$ $\delta / \mathrm{ppm}: 13.82,37.79,108.84,109.65,118.90,119.55$, $120.70,122.22$, 123.20,123.85, 124.38, 124.89, 126.32, $126.75,129.85,134.40,139.29,140.53,180.60 ; \mathrm{MS}(\mathrm{m} / \mathrm{z})$ : $424.10(\mathrm{M}+\mathrm{H})^{+}$

1-(9-ethyl-9H-carbazol-6-yl)-3-p-tolylthiourea (5f) Light yellow colored solid; Yield (65\%); m.p. $143-144{ }^{\circ} \mathrm{C}$; IR $\tilde{v}_{\max } / \mathrm{cm}^{-1}$ : $3366(\mathrm{NH}), 3179(\mathrm{NH}), 2970(=\mathrm{CH}), 1531(\mathrm{C}=\mathrm{S})$; ${ }^{1} \mathrm{H} \mathrm{NMR}\left(\mathrm{CDCl}_{3}\right) \delta / \mathrm{ppm}: 1.44\left(\mathrm{t}, 3 \mathrm{H}, J=6.3 \mathrm{~Hz}, \mathrm{CH}_{3}\right), 2.34(\mathrm{~s}$, $\left.3 \mathrm{H}, \mathrm{ArCH}_{3}\right), 4.35\left(\mathrm{q}, 2 \mathrm{H}, J=6.3 \mathrm{~Hz}, \mathrm{~N}-\mathrm{CH}_{2}\right), 7.18-7.29(\mathrm{~m}, 2 \mathrm{H}$, $\mathrm{ArH}), 7.34-7.48(\mathrm{~m}, 2 \mathrm{H}, \mathrm{ArH}), 7.52-7.69(\mathrm{~m}, 5 \mathrm{H}, \mathrm{ArH}), 7.75-$ $7.95(\mathrm{~m}, 2 \mathrm{H}, \mathrm{ArH}), 8.05(\mathrm{~s}, 1 \mathrm{H}, \mathrm{NH}), 8.25(\mathrm{~s}, 1 \mathrm{H}, \mathrm{NH}) ;{ }^{13} \mathrm{C}$ $\mathrm{NMR}\left(\mathrm{CDCl}_{3}\right) \delta / \mathrm{ppm}: 13.81,37.89,108.84,109.68,118.92$, $119.79,120.59,122.28,123.35,123.80,124.39,124.84$, $126.38,126.64,129.70,135.48,139.39,140.75,180.69 ; \mathrm{MS}$ $(\mathrm{m} / \mathrm{z}): 360.06(\mathrm{M}+\mathrm{H})^{+}$. 
Acknowledgment. V. A. Kadnor is grateful to UGC, WRO, Pune for providing a teacher fellowship under the faculty development programme of the UGC's $12^{\text {th }}$ plan. The authors are also grateful to the Central Instrumentation Facility (CIF), SPPU, Pune for providing the characterization.

Supplementary Information. Supporting information to the paper is attached to the electronic version of the article at: https://doi.org/10.5562/cca3700.

PDF files with attached documents are best viewed with Adobe Acrobat Reader which is free and can be downloaded from Adobe's web site.

\section{REFERENCES}

[1] H. J. Knolker, K. R. Reddy, Chem. Bio. 2008, 65, pp. 1-430. https://doi.org/10.1016/S1099-4831(07)00001-6

[2] A. Kuwahara, K. Nakano, K. Nozaki, J. Org. Chem. 2005, 70, 413-419. https://doi.org/10.1021/jo048472+

[3] K. Thevissen, A. Marchand, P. Chaltin, E. M. K. Meert, B. P. A. Cammue, Curr. Med. Chem. 2009, 16, 2205-2211. https://doi.org/10.2174/092986709788612701

[4] A. Gluszynska, Eur. J. Med. Chem. 2015, 94, 405-426. https://doi.org/10.1016/j.ejmech.2015.02.059

[5] A. Caruso, A. S. Voisin-Chiret, J. C. Lancelot, M. S. Sinicropi, A. Garofalo, S. Rault, Heterocycles, 2007, 71, 2203-2210. https://doi.org/10.3987/COM-07-11113

[6] J. Li, A. C. Grimsdale, Chem. Soc. Rev. 2010, 39, 23992410. https://doi.org/10.1039/b915995a

[7] A. Murat, A. S. Sarac, Prog. Org. Coat. 2009, 66, 337-358. https://doi.org/10.1016/j.porgcoat.2009.08.014

[8] H. J. Jiang, S. Jian, J. L. Zhang, Curr. Org. Chem. 2012, 16, 2014-2025. https://doi.org/10.2174/138527212803251604

[9] G. Yaqub, E. A. Hussain, M. A. Rehman, M. Bateen, Asian J. Chem. 2009, 21, 2485-2520.

[10] R. Joyeeta, J. Amit Kumar, M. Dipakranjan, Tetrahedron 2012, 68, 6099-6121. https://doi.org/10.1016/j.tet.2012.05.007

[11] A. W. Schmidt, K. R. Reddy, H.-J. Knölker, Chem. Rev. 2012, 112, 3193-3328. https://doi.org/10.1021/cr200447s

[12] S. L. Rodriguez, G. E. Zaballos, R. E. Gonzalez, M. L. Testa, A. J. Sepulveda, A. R. Jones, Tetrahedron 2000, 56, 4511-4514. https://doi.org/10.1016/S0040-4020(00)00290-8

[13] R. L. Hudkins, N. W. Johnson, T. S. Angeles, G. W. Gessner, J. P. Mallamo, J. Med. Chem. 2007, 50, 433441. https://doi.org/10.1021/jm051074u

[14] M. Prudhomme, Eur. J. Med. Chem. 2003, 38, 123140. https://doi.org/10.1016/S0223-5234(03)00011-4

[15] L. Ackermann, A. Althammer, Angew. Chem. Int. Ed. 2007, 46, 1627-1629. https://doi.org/10.1002/anie.200603833
[16] I. J. Enyedy, Y. Ling, K. Narcho, Y. Tomita, X. Wu, Y. Cao, R. Guo, B. Li, X. Zhu, Y. Huang, Y. Q. Long, P. P. Roller, D. Yang, S. Wang, J. Med. Chem. 2001, 44, 4313-4324. https://doi.org/10.1021/jm010016f

[17] M. H. Block, S. Boyer, W. Brailsford, D. R. Brittain, D. Carroll, S. Chapman, S. D. Clarke, C. S. Donald, K. M. Foote, L. Godfrey, A. Ladner, P. R. Marsham, J. D. Masters, C. D. Mee, M. R. Donovan, J. E. Pease, A. G. Pickup, J. W. Rayner, A. Roberts, P. Schofield, A. Suleman, A. V. Turnbull, J. Med. Chem. 2002, 45, 3509-3523. https://doi.org/10.1021/jm011125x

[18] J. R. Hiscock, C. Caltagirone, M. E. Light, M. B. Hursthouse, P. A. Gale, Org. Biomol. Chem. 2009, 7, 1781-1783. https://doi.org/10.1039/b900178f

[19] J. B. Shi, W. J. Tang, X. B. Qi, R. Li, X. H. Liu, Eur. J. Med. Chem. 2015, 90, 889-896. https://doi.org/10.1016/j.ejmech.2014.12.013

[20] H. V. Chavan, B. P. Bandgar, L. K. Adsul, V. D. Dhakane, P. S. Bhale, V. N. Thakare, V. Masand, Bioorg. Med. Chem. Lett. 2013, 23, 1315-1321. https://doi.org/10.1016/j.bmcl.2012.12.094

[21] A. M. Vijesh, A. M. Isloor, S. Prashant, K. Sundershan, H. Fun, Eur. J. Med. Chem. 2013, 62, 410-415. https://doi.org/10.1016/j.ejmech.2012.12.057

[22] S. A. H. El-Feky, K. A. Zakeria, A. O. El-Samii Nermine, J. Lashine, A. K. Mohemad, H. K. Thabet, Bioorg. Chem. 2015, 58, 104-116. https://doi.org/10.1016/j.bioorg.2014.12.003

[23] C. B. Sangani, D. C. Mungra, M. P. Patel, R. G. Patel, Chin. Chem. Lett. 2012, 23, 57-60. https://doi.org/10.1016/j.cclet.2011.09.012

[24] O. I. El-Sabbagh, M. M. Baraka, S. M. Ibrahim, C. Pannecouque, G. Andrei, R. Snoeck, A. A. Balzarini Rashad, Eur. J. Med. Chem. 2009, 44, 3746-3753. https://doi.org/10.1016/j.ejmech.2009.03.038

[25] B. Insuasty, A. Tigreros, F. Orozco, J. Quiroga, R. Abonía, M. Nogueras, A. Sanchez, J. Cobo, Bioorg. Med. Chem. 2010, 18, 4965-4974. https://doi.org/10.1016/j.bmc.2010.06.013

[26] P. Przybylski, A. Huczynski, K. Pyta, B. Brzezinski, F. Bartl, Curr. Org. Chem. 2009, 13, 124-148. https://doi.org/10.2174/138527209787193774

[27] J. Janockova, J. Plsikova, J. Kasparkova, V. Brabec, R. Jendzelovsky, J. Mikes, J. Koval, S. Hamulakova, P. Fedorocko, K. Kuca, M. Kozurkova, Eur. J. Pharm. Sci. 2015, 76, 192-202. https://doi.org/10.1016/j.ejps.2015.04.023

[28] S. Thiratmatrakul, C. Yenjai, P. Waiwut, O. Vajragupta, P. Reubroycharoen, M. Tohda, C. Boonyarat, Eur. J. Med. Chem. 2014, 75, 21-30. https://doi.org/10.1016/j.ejmech.2014.01.020

[29] A.G. Blackman, Che-minform. 2005, 24, 1-39. https://doi.org/10.1016/j.poly.2004.10.012 
[30] F. Marchetti, C. Pettinari, R. Pettinari, A. Cingolani, D. Leonesi, A. Lorenzotti, Polyhedron 1999, 18, 30413050. https://doi.org/10.1016/S0277-5387(99)00230-2

[31] D. Guo, K. Li, Y. Li, H. Tan, L. Wang, Chem. Eng. Comm. 2013, 200, 1503-1512.

https://doi.org/10.1080/00986445.2012.756395

[32] A. R. Nixha, A. Ergun, N. Gencer, O. Arslan, M. Arslan, Arch. Physiol. Biochem. 2018, 3, 263-269. https://doi.org/10.1080/13813455.2018.1453523

[33] Y. J. Kim, J. H. Ryu, Y. J. Cheon, H. J. Lim, R. Jeo, Bioorg. Med. Chem. Lett. 2007, 17, 3317-3321. https://doi.org/10.1016/j.bmcl.2007.04.005

[34] A. R. Nixha, M. Arslan, Y. Atalay, N. Gencer, A. Ergun, O. Arslan, J. Enzyme Inhib. Med. Chem. 2013, 28, 808-815. https://doi.org/10.3109/14756366.2012.688040

[35] S. Liu, M. C. Louie, V. Rajagopalan, G. Zhou, E. Ponce, T. Nguyen, L. Green, Bioorg. Med. Chem. Lett. 2015, 25, 1301-1315. https://doi.org/10.1016/j.bmcl.2015.01.042
[36] A. P. Keche, G. D. Hatnapure, R.T. Tale, A. H. Rodge, S. S. Birajdar, V. M. Kamble, Med. Chem. Res. 2013, 22, 1480-1487.

https://doi.org/10.1007/s00044-012-0144-5

[37] V. A. Chornous, M. K. Bratenko, M. K. Vovk, Chem. Heterocycl. Compd. 2006, 42, 1242-1243. https://doi.org/10.1007/s10593-006-0233-9

[38] S Liu, M. C. Louie, V. Rajagopalan, G. Zhou, E. Ponce, T. Nguyen, L. Green, Bioorg. Med. Chem. Lett. 2015, 25, 1301-1305. https://doi.org/10.1016/j.bmcl.2015.01.042

[39] B. Boyan, H. James, P. Judicael, J. Antimicrob. Chemother. 2008, 61, 1295-1301. https://doi.org/10.1093/jac/dkn090

[40] K. H. Rieckmann, G. H. Campbell, L. J. Sax, J. E. Mrema, Lancet, 1978, 311, 22-23. https://doi.org/10.1016/\$0140-6736(78)90365-3 